\title{
英格兰遗产影响评估的经验
}

\section{Experience of English Heritage Impact Assessment}

摘要: 目前, 遗产影响评估已经在国际上一定范围内得到实施, 英格兰是其中一个典型, 它将保护对象扩大到了历史环境范 畴。在可持续发展的大背景下, 英格兰面临不可避免的开发 建设。借助强大的规划系统, 针对开发建设对历史环境产生 的影响进行遗产影响评估。通过遗产影响评估, 国家对历史 环境及其场所环境进行管理, 将与其相关的开发与变化纳入 审批程序, 提交遗产的重要性和场所环境对重要性贡献的研 究, 以及对开发影响大小的评估, 提出缓解措施, 使历史环 境的重要性得以延续。

Abstract: Currently the Heritage Impact Assessment implements within a certain scope in the world. England is one of the typical cases. In the context of sustainable development, faced with the inevitable development, England put Heritage Impact Assessment to a strong planning system, especially in the approval process, for managing the historic environment and the setting. The studies include the importance of heritage and the contribution of the setting, and the evaluation of impact the proposed development on historic environment and the setting. All above must be submit. In order to protect historic environment, mitigation measures are important as well.

关键词：英格兰；历史环境；场所环境；遗产影响评估

Keywords: England; Historic Environment; Setting; Heritage Impact Assessment

上海市设计学 IV 类高峰学科：国际联合实验室开放基金“遗 产影响评估（HIAs）方法及其在历史街区保护管理中的应用研 究” (DB17028)

作者: 冯艳, 博士, 华东理工大学艺术设计与传媒学院, 教师。 叶建伟, 博士, 上海同济城市规划设计研究院, 规划师。 yjwfy_2006@163.com

\section{1 背景}

21 世纪以来, 在可持续发展语境下, 保护和发展的关系再 次得到更深人的理解。近年来, 联合国教科文组织世界遗产委员 会 (UNESCO World Heritage Committee) 收到大量大规模开发建 设、土地利用政策调整和大尺度城市结构变化对世界遗产产生影响 的报告, 这些发展变化对遗产的外观、天际线、视觉焦点等各种 承载了遗产价值的属性造成了影响。为了使国际古迹遗址理事会 (ICOMOS) 和世界遗产委员会能准确评估这些潜在的外部干预, 2009 年, 联合国教科文组织与国际古迹遗址理事会编写了《世界 文化遗产影响评估导则》(Guidance on Heritage Impact Assessments for Culture World Heritage Properties) ${ }^{[1]}$, 将其作为一种文化遗产地 的管理方法推介给遗产保护领域, 并作为文化遗产地针对外部建设 活动进行事前干预的行之有效的新方法向各会员国推广。目前, 遗 产影响评估在国际上一定范围内实施, 英格兰就是其中一个典型。

英格兰将历史保护对象扩大到了历史环境 (historic environment) 范畴。与此同时, 尽管它面临不可避免的开发建设, 但在可持续发展的大背景下, 英格兰借助强大的规划系统进行遗 产影响评估, 对历史环境及其场所环境 (setting) 进行管理, 将与 其相关的开发与变化纳人审批程序, 即所有开发需要提交遗产的 重要性和场所环境对重要性贡献的研究以及开发影响大小的评价, 提出缓解措施, 使历史环境的重要性得以延续。其中英格兰遗产 委员会（English Heritage）是责任保护和促进历史环境所有方面的 法定机构。

\section{2 遗产保护管理}

遗产影响评估是在开发建设管理中贯彻遗产保护管理要求的技 术和方法, 通过规划等实现开发建设实施管理的制度性流程。在遗 产保护管理中, 英格兰遗产影响评估的目的是保护历史环境及其场 所环境的重要性, 使其在开发建设中得以延续。 


\section{1 历史环境 ( historic environment )}

英格兰对历史资源的保护从保护单体建筑发展到历史街 区再到对各项遗产的保护 ${ }^{22}$ 。2000 年之后, 英格兰又将保护 对象从遗产扩展到历史环境。历史环境由提出到确定经历了 时间的历练, 并逐步成为当下保护的主要对象。

(1) 概念与内涵

1994 年，环境部（Department of the Environment）和国 家遗产部（Department of National Heritage）公布了《规划政 策引导 15 : 规划和历史环境》(Planning Policy Guidance 15: Planning and the Historic Environment, 简称 PPG15) ${ }^{[3]}$, 历史 环境 ${ }^{1}$ 作为正式术语出现。

1997 年, 英格兰遗产委员会在《维护历史环境 : 面向 未来的新视角》(Sustaining the Historic Environment: New Perspectives in the Future）中提出，人们应该珍视那些被明确 公布的文化遗产, 同时应清楚认识到日常环境塑造着地方的 特定性和身份 ${ }^{[4]}$ 。可见, 英格兰遗产委员会通过区别遗产对 象与日常环境来界定历史环境。2000 年, 英格兰遗产委员 会发布《地方的力量 : 历史环境的未来》(Power of Place: the Future of Historic Environment), 明确 “历史环境是人们在其 生活的地方留下的遗存, 历史环境就在人们身边。我们生活 的城镇、乡村都是由各个时期人类活动累积叠加而成。每一 代人都留下了自己的印记, 每个人在进行关于未来发展的各 种决策时, 都应充分考虑地方的文脉特征。环境不可替代, 一旦失去, 将永远失去。” ${ }^{[5]} 2001$ 年, 文化、媒体与体育部 (DCMS: Department of Culture, Media and Sport) 发布《历 史环境: 未来的力量》(The Historic Environment : A Force for our Future), 强调历史环境对社区的意义，应全力释放英 格兰历史环境的潜能, 提升生活品质 ${ }^{[6]}$ 。

2008 年，英格兰遗产在《历史环境可持续管理的保 护原则、政策和导则》(Conservation Principles, Policies and Guidance）中第一次将历史环境明确定义为人与场所之间长 时间相互作用所产生的周围环境的总和，包括过去人类活动 的所有物质遗存 ${ }^{[7]}$, 其中的重要部分称之为遗产。

在基本确定了“历史环境” 的概念之后, 2010 年国家 社区与地方政府部 (Communities and Local Government) 颁布了《规划政策声明 5 : 历史环境规划》(Planning Policy Statements 5: Planning for the Historic Environment, 简称 PPS5)。 它肯定了历史环境对文化、社会和经济的价值, 并提出规划 在保护遗产和管理历史环境上发挥了主要作用，政府希望对 历史环境的管理通过规划系统来实现，使历史环境更好地保
存下去 ${ }^{[8]}$ 。与 PPS5 相配合, 2010 年社区与地方政府部、英 格兰遗产委员会联合文化、媒体与体育部共同颁布《历史环 境规划实践指导》(Historic Environment Planning and Practice Guide), 提出 12 项历史环境政策, 强调预先申请的重要性 ${ }^{[10]}$ 。 2012 年 3 月, 社区和地方政府部发布了最新的规划政 策《国家规划政策框架》(NPPF: The National Planning Policy Framework) ${ }^{[11 !}$, 历史环境及相关的管理在 NPPF 中得到了 延续。

至此, 经过长达 18 年的发展, 英格兰的历史保护范畴 从传统遗产扩大到人们的生活环境。

（2）历史环境档案 (Historic Environment Records)

一旦我们对历史环境有了比较清楚的认识, 第二步就是 管理历史环境。PPS5 中定义历史环境档案是为公共利益及 公共使用提供有关特定地理区域历史环境的全方位资源, 包 含了可链接地理信息系统的数据库和相关参考资料。

历史环境档案通常由统一的部门或上一级地方部门, 或 国家公园部门主管, 在大伦敦地区由英格兰遗产委员会主管。 地方规划部门应维持并参考历史环境档案, 应利用这些信息 对遗产的类型、数量、分布、重要性和状况进行评估, 对遗 产对当下及未来环境的作用进行评估 ${ }^{[8]}$, 对可能存在的遗产 进行预测。历史环境档案提供了综合特定区域的历史环境信 息和证据, 是遗产影响评估的基础资料来源。

\section{2 场所环境 ( setting )}

(1) 概念与内涵

PPS5 提出了场所环境这一概念, 指遗产所能依附 (experienced) 的周边环境, 如土地、海洋、建筑物、构筑 物、外观、景观、天际线及重要景点, 涉及物质和非物质范 畴。它的范围是不固定的, 随着遗产和周围环境的发展而改 变。无论以何种方式存在, 遗产都有场所环境。场所环境并 没有固定的空间边界, 其视觉要素十分重要, 但也受噪音、 灰尘及震动等其他环境因子的影响。

（2）遗产的重要性和场所环境

场所环境的要素可能对遗产重要性产生积极、消极或中 立的 (neutral) 影响 ${ }^{[12]}$ 。

遗产的重要性不仅来源于其物质特征和历史结构, 还 来自场所环境。管理好遗产周边环境的发展变化, 可以创造 更高质量的生活场所。无论何时, 场所环境可以提供给人们 欣赏遗产重要性的机会。费尔菲尔德的圣托马斯贝克特教堂 (St Thomas a Beckett at Fairfield)（图 1) 孤立的场所环境,

(1) PPG15 中规定历史环境包括登录建筑 (Listed Buildings)、保护区 (Conservation Areas)、世界遗产 (World Heritage Sites)、历史公园和花园 (Historic Parks and Gardens)、历史战场（Historic Battlefields）、更广泛的历史景观（the Wider Historic Landscape）。 
证明罗姆尼湿地（Romney Marsh）历史聚居地的边缘特征, 因此对其重要性产生较大影响。

遗产的场所环境可以增强其重要性, 比如北安普敦郡 考特斯布鲁克庄园 (Cottesbrooke Hall) 周边园林的设计 (图 2); 又如威尔士大教堂 (Wells Cathedral) 看似偶然的美, 源于和谐的尺度、设计和材质 (图 3)。历史战争遗址也存 在场所环境, 如布洛希思之役 (Blore Heath battlefield) 遗址

(图 4)。城镇景观包括很多遗产, 有着相互交叉和重叠的场 所环境, 如托特尼斯古镇 (historic town of Totnes)（图 5)。

不良的设计或开发会削弱遗产与场所环境之间的关系。 汉普郡 (Hampsuire) M3 公路的施工限制了场所环境, 交 通和噪音对哈特利温特尼 (Hartley Wintney) 老谷仓的遗产 重要性造成了叠加影响, 严重阻碍了一系列可行的再利用 改造。最终谷仓沦为车展展厅, 未能充分体现出它的历史 价值（图 6)。

\section{3 开发建设管理}

\section{1 开发建设}

为使开发建设能更积极地融合到历史环境保护中, 不仅 需要对历史环境有清楚的理解, 更需要评估开发建设是否适
宜, 以及开发建设如何应对特定情况。国家层面的立法和指 导提出了用于评估开发建设对历史环境影响的可接受性或不 可接受性的总原则。从 PPG15 到 PPS5, 再到 NPPF, 英格兰 的实践都传达出对遗产管理与开发建设两者关系的关注。

1994 年的 PPG15 指出要通过规划体系来定义环境的可 变化能力, 提出当新开发方案提出之后, 需要评估它们对历 史环境的影响。

2008 年《历史环境可持续管理的保护原则、政策和导 则》中明确指出, 在开发管理选择保护措施, 或协调保护其 他利益时, 遗产影响评估可以用来比较预测到的行动替代路 线（包括还没有进行的任何行动）对场所环境的影响, 以确 定最优的解决方案。

2010 年 PPS5 提出, 要保护遗产就要对遗产及其周边的 开发进行管理。PPS5 虽然并未明确提出 “遗产影响评估”, 但在开发管理中明确指出, 开发项目在申请时, 申请者应描 述遗产的重要性和场所环境对重要性的贡献, 充分理解开发 计划对遗产重要性的潜在影响。在考虑影响时, 申请者应咨 询相关的历史环境档案, 必要时使用适当的专业知识对遗产 本身进行评估。相关影响评估信息有必要在设计和使用声 明 ${ }^{1}$ 中阐述, 作为解释设计概念的一部分 ${ }^{[8]}$ 。

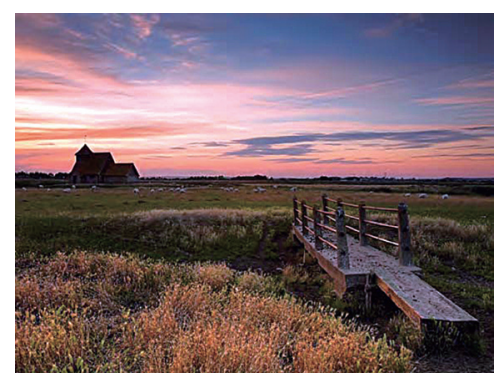

图 1 圣托马斯贝克特教堂 资料来源 : 参考文献 [12]

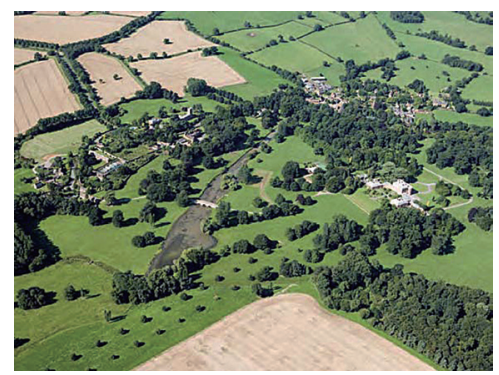

图 2 考特斯布鲁克庄园周边的园林 资料来源 : 同图 1

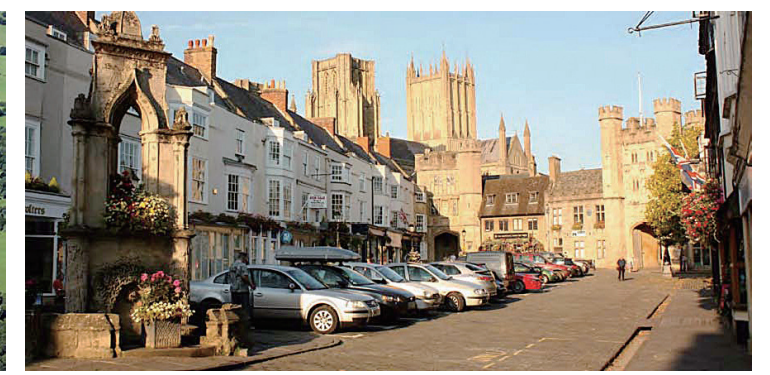

图 3 威尔士大教堂 资料来源 : 同图 1

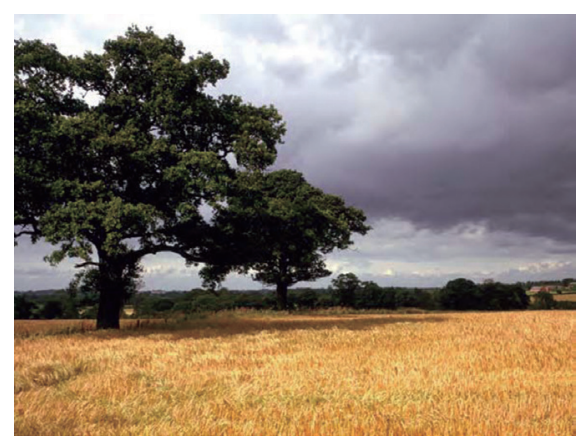

图 4 布洛希思之役遗址 资料来源 : 同图 1

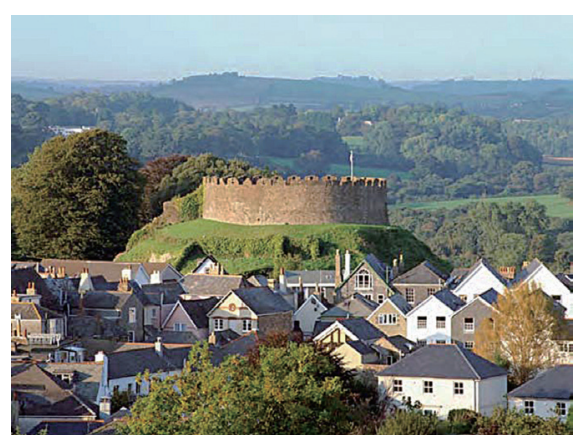

图 5 托特尼斯古镇 资料来源：同图 1

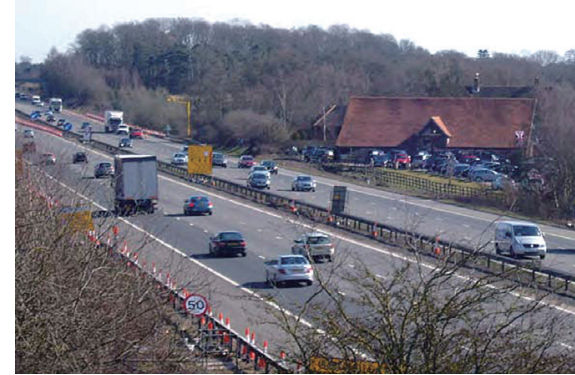

图 6 汉普郡哈特利温特尼老谷仓 资料来源 : 同图 1

(1) 设计和使用声明 (Design and Access Atatement) 是一个简短的报告, 与规划许可一起并支持规划许可。它为申请者提供框架来解释开发计划是 如何适应此地块和它的场所环境, 并论证开发是可以满足预期使用者的 ${ }^{[20]}$ 。 
NPPF 在延续 PPS5 的思路上进一步加强对遗产周边开发 的管理, 对遗产影响评估提出了指导。NPPF 建议应给予遗 产保护以最大的重视, 遗产越重要, 决策时越应重视。它明 确了遗产是 “不可替代的”, 遗产所经受的任何损害都需要 “明确并令人信服的理由” ${ }^{[11]}$ 。

\section{2 相关部门}

遗产影响评估涉及英格兰遗产委员会和地方政府。其全 程都有英格兰遗产委员会的参与 ; 而地方政府在遗产保护中 的作用也颇为关键, 它的重要职能之一就是平衡开发建设与 遗产保护之间的关系, 评估新建设活动对地方环境带来的益 处。地方政府通过地方规划（Local Planning）确定不适当的 开发 ${ }^{[13]}$, 评估某些可能对历史环境产生影响的开发区域。地 方规划包括地方发展框架（Local Development Frameworks） (1) 和开发控制 (Development Control), 由地方规划当局制定。

\section{3 遗产影响评估}

遗产影响评估是规划许可的支撑信息和前期研究，而地 方发展框架中的地方发展规划（Local Development Plans）则 对遗产影响评估进行指导。

土地所有者或开发商计划改变土地使用性质, 必须向地 方规划部门（LPA）申请开发许可。LPA 在作出决定之前需 要遵循审批流程, 这一过程以及相关规划文件被称为开发控 制或开发管理（development management）。地方规划部门决 策时, 应设法识别和评估可能受到相关开发影响的历史环境 重要性的所有要素 (包括开发影响的遗产场所环境)。如果 从申请和相关文件中不能充分理解开发计划对任何遗产重要 性造成影响的程度, 那么地方规划部门不应批准申请 ${ }^{[81}$; 如 果证实开发将对遗产产生负面影响或伤害, 或使遗产环境恶 化, 也不给予许可; 如果地方规划部门认为开发造成了遗产 重要性的损害, 在采取合理的措施之前将不能继续开发计划。

\section{4 遗产影响评估的主要内容}

\section{1 方法来源}

英格兰的遗产影响评估方法框架来自环境影响评估（EIA： Environmental Impact Assessment）。此外, 欧盟指令（European Directives）中的相关内容同样适用于遗产影响评估 ${ }^{[14]}$ 。

2007 年高速公路局（The Highways Agency）发布的《道 路和桥梁设计手册》(Design Manual for Roads and Bridges) ${ }^{[15]}$
为决策过程提供了支持, 为评估道路项目可能对文化遗产重 要性产生的影响提供了全面指导。文化遗产影响评估的方法 在其中单独成章, 指明文化遗产, 连同其场所环境, 可以被 视为一个整体来进行评估。地方规划部门应该在发展规划中 确定文化遗产价值。

手册中指出评估有三个层次 : 确定范围、简化评估、详 细评估。手册中提出了影响的重要性矩阵 (表 1), 成为日 后遗产影响评估的主要方法。该评估方法已多次被用于评估 巨石阵项目的影响, 使它通过了规划系统和公开咨询 (图 7)。 2009 年《巨石阵游客设施及交通方案环境声明》(Stonehenge Visitor Facilities and Access Scheme Environmental Statement) ${ }^{[16]}$ 阐 述了遗产委员会实施巨石阵的游客设施和交通方案的计划, 以改善对游客的供给和完善巨石阵的整体印象。此声明作为 规划申请的支撑信息 ${ }^{[17]}$, 在提出规划申请时一并交给审批 部门。

巨石阵是英格兰最重要的遗产之一, 吸引全世界的游客 前来参观。然而, 不适当的游客设施建设以及对巨石阵历史

\section{表 1 影响的重要性矩阵}

\begin{tabular}{l|l|l|l|l|l}
\hline \multirow{2}{*}{ 价值 } & \multicolumn{5}{|c}{ 影响量级 } \\
\cline { 2 - 6 } & 没有变化 & 可忽略变化 & 微小变化 & 中等变化 & 大 (主要) 变化 \\
\hline 极高 & 中立 & 轻微 & 中等/大 & 大/非常大 & 非常大 \\
\hline 高 & 中立 & 轻微 & 中等/轻微 & 中等/大 & 大/非常大 \\
\hline 中等 & 中立 & 中立/轻微 & 轻微 & 中等 & 中等/大 \\
\hline 低 & 中立 & 中立/轻微 & 中等/轻微 & 轻微 & 轻微/中等 \\
\hline 可忽略 & 中立 & 中立 & 中等/轻微 & 中立/轻微 & 轻微 \\
\hline
\end{tabular}

资料来源 : 参考文献 [15]

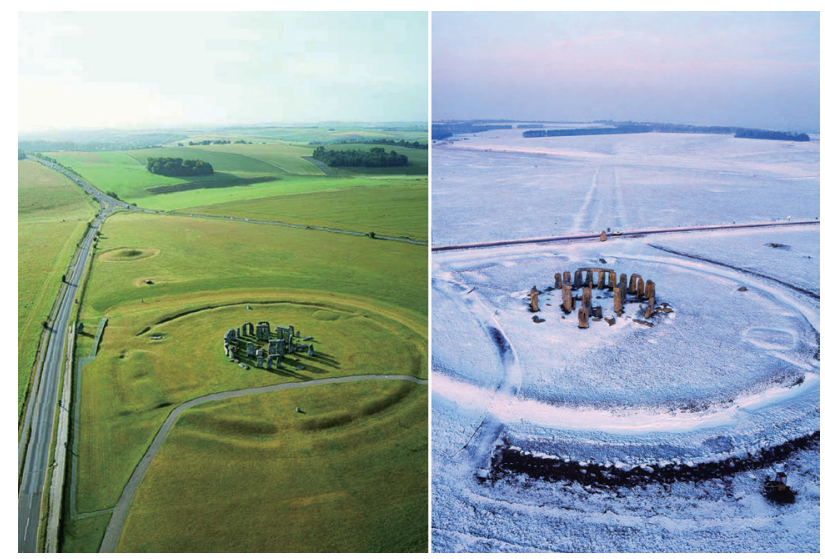

图 7 巨石阵

资料来源 : 参考文献 [16]

(1) 2004 年的《规划和强制收购法》(Planning and Compulsory Purchase Act) 提出地方发展框架，取代了之前的地方规划（Local Plans)。地方发展 框架中包括: 社区参与声明 (Statement of Community Involvement)、发展规划文件 (Development Plan Documents) 和补充规划文件 (Supplementary Planning Documents)。 
景观的损害是遗产管理部门长久以来关注的重点。《巨石阵 游客设施及交通方案环境声明》根据《1999 年英格兰和威 尔士城镇规划（环境影响评估）条例》(Town and Country Planning [Environmental Impact Assessment] England and Wales Regulations 1999), 主要采用环境影响评估的过程和方法 ${ }^{[18]}$, 对巨石阵的游客设施和交通方案计划对巨石阵的影响进行评 估。环境声明预测了开发计划对该地区的影响, 提出措施来 避免、减轻、补救任何潜在的重大负面影响, 同时加强游客 设施, 完善交通方案计划的有利影响措施。

\section{2 主要内容}

评估开发对遗产及其场所环境产生的影响, 需要系统、 阶段性的方法, 这是设计导则或环境声明的基础。2011 年英 格兰遗产委员会在探讨场所环境的开发管理时, 基于该方法 提出了遗产影响评估的主要内容。

（1）识别出受到影响的遗产及其场所环境

在申请前或范围确定阶段, 地方规划部门应该指出开发 计划对特定遗产场所环境是否存在潜在影响, 划定开发计划 周边的合理研究区域, 对该区域内的遗产及其场所环境进行 识别。

在划定开发计划周边合理研究区域时, 建议使用视觉影 响分区（zone of visual influence）或理论可视分区（zone of theoretical visibility) 方法, 以便更好地确认开发计划对遗产 和场所环境的影响。

英格兰遗产影响评估注重视觉等方面的影响, 视觉影响 可能是某些开发主要的或唯一需要评估的影响, 可以借鉴环 境影响评估中的景观和视觉影响评估 (Landscape and Visual Impact Assessment) ${ }^{[18]}$ 。英格兰遗产委员会 2012 年出版《视 图中的历史》(Seeing the History in the View) ${ }^{[19]}$, 对历史资源 的视线, 通廊进行了分析。观景地是一个空间范围, 可能有 完整的边界, 也可能没有, 其中包括了评估点和一系列视点, 评估点应该是其中的最佳观赏视点（图 8)。

（2）评估场所环境对遗产重要性的贡献、方式和程度

确定场所环境对遗产重要性的作用大小。主要从场所 环境的潜在属性分析人手, 来阐明其对遗产重要性的贡献。

历史环境档案是评估的重要资料基础, 为认知遗产更广 泛的景观环境背景和遗产自身提供信息。

（3）评估拟开发项目对重要性的影响

确定开发对场所环境影响的范围, 评估开发对遗产重要 性有害或有利的程度, 可以从研究开发项目的关键属性人手。

根据详细程度的不同, 应选取最关键的属性进行分析。 在评估早期阶段, 就可以对开发活动进行大致利弊分析, 通 过分析项目的具体实施内容, 总结出评估应主要关注的方面。
（4）探寻最大限度增强遗产重要性、避免或最小化损害 的途径

减少损害的方法包括开发或要素的重新布局、设计改变、 设立长期有效的视觉和听觉屏障, 以及规划条件或法定协议 约束下的管理措施等。通过良好的设计降低或者避免损害, 或增强遗产价值重要性。

（5）作出决策并形成文件, 检测成果

决策的每个步骤都应用通俗而确定的说明来形成文件。 一旦确定开发对遗产的场所环境产生影响, 在后续采取措施 来强化重要性或减轻损害时, 就可以回顾其他成功案例, 汲 取相关的经验教训。

以上五个步骤是英格兰遗产影响评估的一般性内容, 在 评估过程中, 每个步骤都有具体的工作内容予以落实（表2）。

\section{5 结语}

遗产保护不是阻止变化, 而是帮助管理无法规避的变化 过程。它树立了开发商和土地所有人的信心, 即遗产能为变 化作积极贡献, 而不是完全约束它 ${ }^{[22]}$ 。

当前, 可持续发展是发展主题。英格兰遗产保护管理借 助规划管理体系, 并具有自身的特点。虽然在国家层面没有 明确的遗产影响评估相关条例, 但在对场所环境应对发展变 化的管理中明确了相应的方法, 形成了较为独特的、完整的 遗产影响评估体系（图 9)。

英格兰将遗产保护对象扩大到历史环境范畴, 从而扩展 了保护的范围。它管理的核心是重要性, 通过评估重要性来 对历史环境定级, 归人历史环境档案, 为遗产影响评估提供 基础资料。

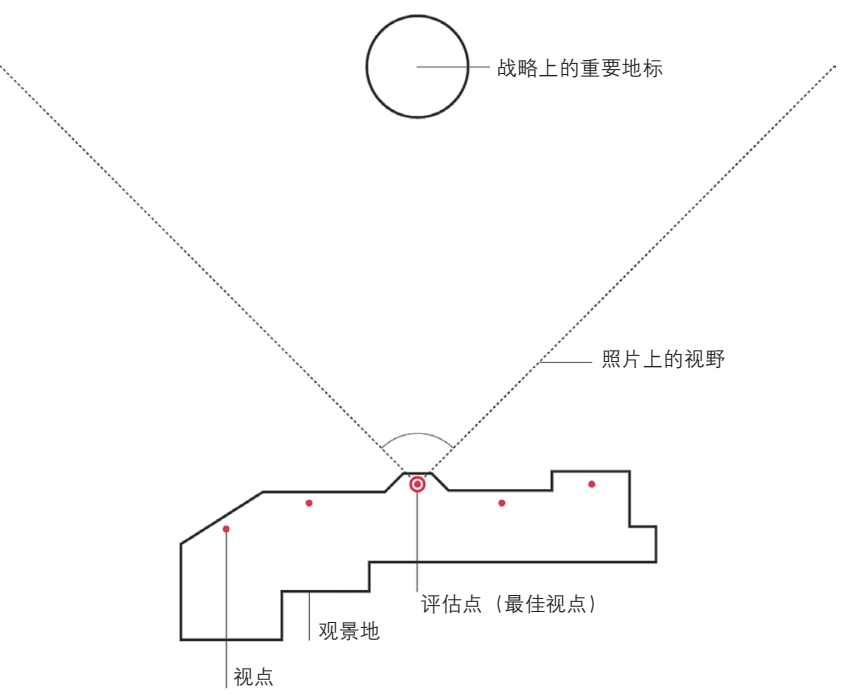

图 8 评估点、视点、观景地

资料来源 : 参考文献 [19] 


\section{表 2 英格兰遗产影响评估具体内容}

\section{第一步 : 识别出受到影响的遗产及其场所环境}

在较广区域内确定遗产 (无论是指定遗产还是非指定遗产) ; 识别出遗产的场所环境 (无论是直观环境还是引申环境); 基于地形、景观特色、视域、土地利用、 公共空间及交通模式建立 “理论视觉影响区” 及 “视觉影响区”; 识别出受到开发项目影响的遗产 (受体); 描述受到拟开发项目影响的遗产重要性

\section{第二步 : 评估场所环境对遗产重要性有无贡献, 以何种方式, 达到何种程度}

\begin{tabular}{|c|c|}
\hline 考虑遗产的物质环境 & $\begin{array}{l}\text { 地形 ; 其他遗产 (包括建筑物、构筑物、景观、区域或考古遗存); 周边的街道景观、景观和空间的清晰度、尺度和纹理 ; 规则式设 } \\
\text { 计 ; 历史材料和外观 ; 土地利用 ; 绿色空间、树木和植被 ; 开敞空间、围墙和边界; 功能性的关系和交流 ; 历史与发展流变 ; 整体性; } \\
\text { 例如土壤化学和水文等地质问题 }\end{array}$ \\
\hline 考虑遗产的感知 & $\begin{array}{l}\text { 周边景观或城镇景观的特色; 以遗产为中心的一系列视图; 作为焦点的视觉支配、显著性或作用; 与其他历史及自然名胜预留的视 } \\
\text { 线通廊；噪音、震动及其他污染源或干扰物；宁静、偏远、荒芜; 围和感、隔离感与私密性 ; 动力和活力; 可达性、通透性、交通 } \\
\text { 模式; 面向公众进行解读与推广的程度; 遗存的场所环境的稀有性 }\end{array}$ \\
\hline $\begin{array}{l}\text { 考虑遗产的关联属性 } \\
\text { (associative attributes) }\end{array}$ & 遗产之间的相互联系；文化联系；著名艺术；传统 \\
\hline \multicolumn{2}{|r|}{ 第三步 : 评佔拟开发项目对重要性的影响 } \\
\hline 开发项目的位置和选址 & 邻近遗产 ; 程度 ; 在地形中的位置 ; 在某些位置上对遗产在物质空间或视觉上产生影响的程度 ; 观景位置 \\
\hline 开发项目的造型和外观 & $\begin{array}{l}\text { 显著、突出、惹人注目 ; 对遗产造成的干扰 ; 规模、尺度和体量 ; 比例 ; 视觉渗透性 (包括能看到的所有区域); 材料（质地、色彩、 } \\
\text { 反光等); 建筑形式和设计 ; 交通或活动 ; 每天或季节的变化 }\end{array}$ \\
\hline 开发项目的其他影响 & $\begin{array}{l}\text { 建设环境和空间的改变; 天际线的改变; 噪声、气味、振动、灰尘等; 灯光效应; 总体特色的变化; 公众可达性、使用或舒适度的改变; } \\
\text { 土地使用、土地覆盖, 树木植被的改变 ; 考古环境、土壤化学或水文的变化; 沟通、可达性、渗透性的变化 }\end{array}$ \\
\hline 开发的持久性 & 预期周期 / 暂时 ; 循环; 可逆 \\
\hline 开发的长期和间接影响 & 所有权的改变 ; 经济和社会的活力 ; 公共使用和社会活力 \\
\hline
\end{tabular}

移除或重建不协调的建筑及外观; 用新的和更和谐的形式替代 ; 恢复或展示业已消失的历史特色; 引人全新特色加强遗产的公众观赏性 ; 引人全新视图增进公众 对遗产的感知

第五步 : 作出决策并形成文件，检测成果

资料来源 : 作者整理

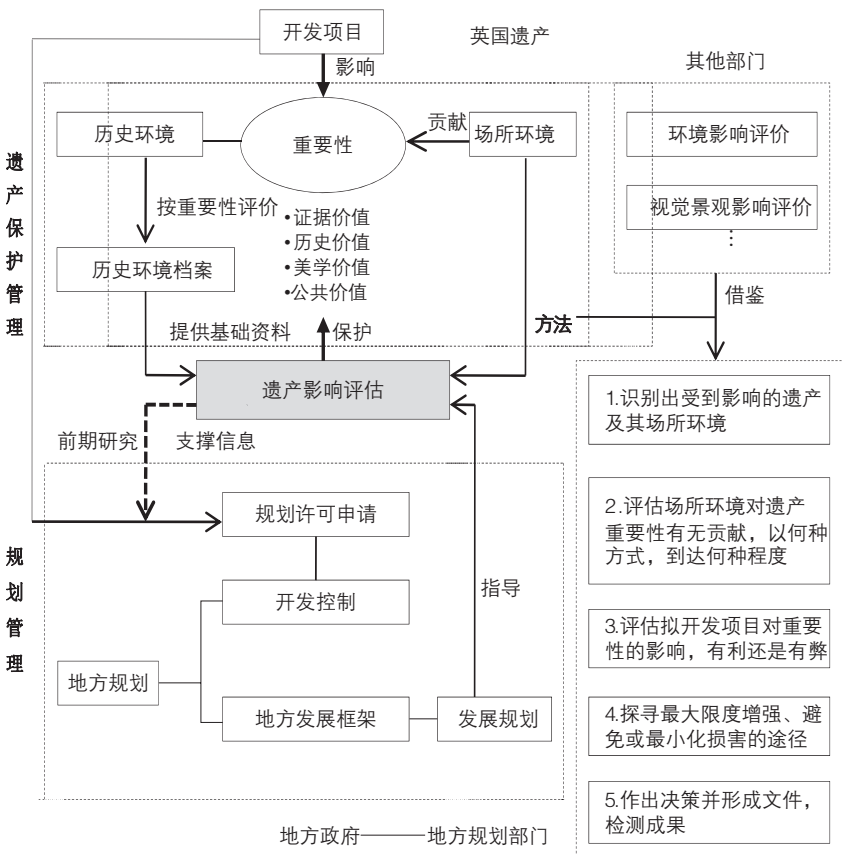

图 9 英格兰遗产影响评估体系 资料来源 : 作者绘制
场所环境是遗产所依附的周边环境。遗产的重要性不仅 来源于其物质特征和历史结构, 还来自场所环境。管理好遗 产周边环境中的发展变化, 可以创造更高质量的生活场所。 因此, 英格兰遗产委员会在借鉴了环境影响评估的方法后, 将其应用于对场所环境应对发展变化的管理, 提出了评估的 五个主要步骤。

为保障遗产影响评估的顺利进行, 将与历史环境相关的 开发与变化纳人规划许可申请程序, 由地方规划和遗产管理 部门共同负责。在开发前的许可申请阶段, 须评估开发项目 对历史环境的影响, 提交遗产的重要性和场所环境对重要性 贡献的研究以及开发影响大小的评估, 提出缓解措施, 同时 在整个过程中, 向英格兰遗产委员会咨询, 使历史环境的重 要性得以延续。

值得一提的是, 英格兰遗产影响评估十分注重视觉方面 的影响, 在方法中借鉴了景观视觉影响评估 (LVIA) 的内容, 与对场所环境的管理结合起来, 在评估过程中划定理论视觉 分区, 进一步加强了遗产影响评估的有效性。 


\section{参考文献}

[1] Guidance on Heritage Impact Assessments for Culture World Heritage Properties[R]. ICOMOS. 2011.

[2] 马尔科姆 - 库珀. 英格兰历史城市环境的管理 [J]. 上海城市规划, 2004(6): 32-38

[3] Department of the Environment, Department of National Heritage. Planning Policy Guidance: Planning and the Historic Environment, PPG15[R]. 1994.

[4] English Heritage. Sustaining the Historic Environment: New Perspectives in the Future. 1997.

[5] English Heritage. Power of Place: The Future of Historic Environment[R]. 2000 .

[6] DCMS. The Historic Environment: A Force for our Future. 2001.

[7] English Heritage. Conservation Principles Policies and Guidance for the Sustainable Management of the Historic Environment[R]. 2008.

[8] CLG: Communities and Local Government. Planning Policy Statement 5: Planning for the Historic Environment[R]. 2010.

[9] Department for Culture, Media and Sport. Government's Statement on the Historic Environment for England[R]. 2010.

[10] Department for Communities and Local Government, Department for Culture, Media and Sport, English Heritage. Historic Environment Planning and Practice Guide[R]. 2010.
[11] CLG. The National Planning Policy Framework[R]. 2012.

[12] English Heritage. The Setting of Heritage Assets[R]. 2011.

[13] English Heritage. Historic Environment Good Practice Advice in Planning Note 1: The Historic Environment in Local Plans[R]. 2014.

[14] Vakhitova T V. Heritage Impact Assessment: Experience from the UK[C] // IAIA11 Conference Proceedings: Impact Assessment and Responsible Development for Infrastructure, Business and Industry 31st Annual Meeting of the International Association. 2011.

[15] The Highways Agency. Design Manual for Roads and Bridges: Volume 11 Section 3 Culture Heritage[R]. 2007.

[16] English Heritage, Chris Blandford Associates. Stonehenge Visitor Facilities and Access Scheme Environmental Statement[R]. 2004.

[17] English Heritage. Stonehenge World Heritage Site Management Plan 2009[R]. 2009.

[18] IEMA \& Landscape Institute. Guidelines for Landscape and Visual Impact Assessment, GLVIA3[R]. 2013.

[19] English Heritage. Seeing the History in the View[R]. 2012.

[20] Planning Portal. Design and access statements[EB/OL]. [2013-12]. http://www.planningportal.gov.uk/planning/applications/howtoapply/ whattosubmit/designaccess.

(本文编辑：胡文娜)

\section{$>>$ 译者随笔 叶齐茂}

\section{街头巷尾的美好生活需要}

我们的美好生活需要数不胜数, 街头巷尾的美好生活需要 应该是我们的美好生活需要之一。实际上, 街头巷尾的美好生 活需要也很多, 清洁、安全、美丽是应该得到满足的需要, 当然, 有个地方可以随意走一走、站一站、坐一坐、说一说话, 还不 担心被小汽车、摩托车、电动车、自行车撞上, 同样是应该让 人民得到满足的美好生活需要。这些美好生活需要原本得到过 满足, 50 后和 60 后恐怕都亲历过那个时代。可是, 随着机动 车的增加, 机动车道的扩宽, 允许使用靠近马路牙子的路边和 人行道停车, 允许在小区内部狭窄的道路上、花坛里、任何边 边角角的地方停车, 我们可以走一走、站一站、坐一坐、说一 说话的地方减少了, 变窄了、不安全了。这不仅是城市街头巷 尾的问题, 富裕的乡村村庄同样如此。机动车及其相关基础设 施的不平衡发展与我们街头巷尾的美好生活需要发生了矛盾, 产生了摆在我们面前的新时代的城市设计问题, 尤其是如何通 过城市设计满足人们精神生活需要的问题。

物质生活丰富了, 精神生活的空间被挤占了, 这个矛盾在
西方发达国家曾经出现过。尽管它们的这种街头巷尾问题在规 模和严重程度上都不能与我们相比, 但是, 即使那样, 他们的 城市规划师和设计师就已经大声疾呼了, 他们从 20 世纪 60 年 代开始, 就一直在对这种街头巷尾的问题展开深入研究, 探索 人们的街头巷尾生活需要, 物质的和精神的, 然后提出种种设 计方案。怀特的《城市: 重新发现市中心》(上海译文出版社, 2018）和《小城市空间的社会生活》（上海译文出版社，2016）, 堪称研究街头巷尾美好生活需要的经典, 城市设计思想的精㰐。 信手拈来几段, 以飨读者。

“无论街头生活有什么意义, 最让人着迷的街头生活都发 生在人们的互动交流之中。”

“就人与人之间的交流而言, 大城市可能明显比小城市友 善, 这种比较不仅仅是就人与人之间交流的绝对数字而言, 还 包括人与人之间交流的整体比例。对比之下, 在小城市里, 我 们看到, 人与人之间的交流比较少, 不断说再见, 又不断继续 下去的那种谈话现象比较少, 街头聚会比较少, $100 \%$ 的谈话比 较少, 供 $100 \%$ 谈话的地方比较少……然而, 就人与人之间交 流的频率而言, 大城市的街头巷尾明显比小城市要活跃一些。”

(下转 134 页) 